\title{
Lifetimes of excited electrons in Ta: Experimental time-resolved photoemission data and first-principles GW+T theory
}

\author{
V. P. Zhukov, ${ }^{1}$ O. Andreyev, ${ }^{3}$ D. Hoffmann, ${ }^{3}$ M. Bauer, ${ }^{3}$ M. Aeschlimann, ${ }^{3}$ E. V. Chulkov, ${ }^{1,2}$ and P. M. Echenique ${ }^{1,2}$ \\ ${ }^{1}$ Donostia International Physics Center (DIPC), 20018 San Sebastián, Spain \\ ${ }^{2}$ Departamento de Física de Materiales and Centro Mixto CSIC-UPV/EHU, Facultad de Ciencias Químicas, UPV/EHU Apdo 1072, \\ 20018 San Sebastián/Donostia, Spain \\ ${ }^{3}$ Department of Physics, University of Kaiserslautern, 67663 Kaiserslautern, Germany
}

(Received 15 April 2004; published 21 December 2004)

\begin{abstract}
Time-resolved two-photon photoemission spectroscopy and first-principles GW and GW+T theories have been used to study excited electron lifetimes in tantalum. The GW+T approach includes evaluation of the lowest self-energy term of the many-body perturbation theory in the GW approximation and higher terms in the $T$-matrix approximation. The GW+T calculated lifetimes are in good agreement with the measured lifetimes at excitation energies above $1.6 \mathrm{eV}$. At lower energies, a slightly worse agreement between theoretical and experimental data is obtained which we refer to as the influence of cascade processes.
\end{abstract}

DOI: $10.1103 /$ PhysRevB.70.233106

PACS number(s): 71.15. $-\mathrm{m}, 78.47 .+\mathrm{p}, 79.60 .-\mathrm{i}$

The dynamics of hot electrons is fundamental to the understanding of many phenomena such as chemical reactions on surfaces, transport, molecular-surface interactions, etc. The knowledge of relaxation times of electron excitations, and consequently the escape-depth length of excited electrons, is important for the interpretation of photoemission spectra, Auger spectra, and low-energy electron diffraction.

Of the experimental methods developed for the study of relaxation times, the most suitable is time-resolved twophoton photoemission spectroscopy (TR-2PPE), ${ }^{1}$ which allows the measurements directly in the time domain. A series of TR-2PPE experiments have been performed for nonmagnetic metals, ${ }^{2-5}$ ferromagnetic metals, ${ }^{6,7}$ and high- $T_{c}$ superconductors. ${ }^{8}$ It has been shown that the most important factor that determines the relaxation times in TR-2PPE experiments is the inelastic electron-electron scattering. Some other processes also affect the relaxation time of a depleted state. One of them, increasing the relaxation time, is the effect of refilling the depleted state with the cascade electrons from higher excited states. Another is a transport effect that reduces the relaxation time due to the diffusion of electrons from irradiated spots on the surface into the bulk.

On the theoretical side, several methods have been developed for the evaluation of excited electron lifetimes, including semiempirical approaches based on scattering theory ${ }^{10,11}$ and first-principles approaches based on the self-energy formalism of many-body perturbation theory (MBPT). ${ }^{12}$ A number of calculations have been performed for the lifetimes of excited electrons in simple, noble, and transition metals. ${ }^{13,14}$ Such methods take into account only inelastic electronelectron scattering, so the comparison of theoretically calculated lifetimes with experimental relaxation times meets with essential difficulties. Besides, the theoretical methods contain a number of intrinsic approximations. Hitherto the best theoretical results have been obtained by means of the firstprinciples GW approach (GWA), which incorporates for the self-energy only the first exchange term of the MBPT. It is well known that in systems with efficient short-range screening (e.g., transition metals), the high-order terms of MBPT can be important. However, in a recent work, Ref. 15, a first-principles $\mathrm{GW}+\mathrm{T}$ method for lifetime calculations outside GWA was discussed. Experimental data on relaxation times in transition metals, except noble metals, are also rare: to our knowledge, only relaxation times in Rh (Ref. 3) have been measured. So new measurements and efforts at calculations with more powerful theoretical methods are desirable.

In this Brief Report, we discuss the application of the TR-2PPE technique for studying relaxation times of electronic excitation in Ta. We also discuss the theoretical lifetimes calculated by means of a first-principles $\mathrm{GW}+\mathrm{T}$ approach which includes, together with the traditional GW term, the $T$-matrix term of MBPT. This discussion shed light on the importance of the above-mentioned experimental (transport and cascade processes) and theoretical (high-order terms of MBPT) factors relating to relaxation times.

In TR-2PPE experiments, the first (pump) pulse populates an intermediate state located between the Fermi edge and the vacuum level. A second (probe) pulse, that is delayed by a time $\tau$, then transfers the remaining population to the detected state above vacuum level. A control of the temporal delay $\tau$ between pump and probe pulses in the femtosecond time scale enables information about the decay dynamics of the excited electrons in the intermediate states. We used pump and probe photon pulses with equal energy and intensities but with crossed polarization in order to minimize the coherent effect between them. The raw data of a TR-2PPE measurement exhibit the two-pulse correlation trace at a definite kinetic energy, where the shape of the trace contains information about pulse width of the two laser pulses as well as population decay time (and hence inelastic lifetime) of the intermediate state. In order to extract the intermediate state lifetime, we applied a MATLAB-based fitting routine which uses a least-squares fit algorithm. This algorithm is based on a quantum-mechanical approach within the density-matrix formalism. ${ }^{16}$ In this formalism, the two-photonphotoemission process is described by an electronic threelevel system interacting with the electromagnetic field of the two laser pulses. The temporal evolution of the density ma- 
trix is usually treated by the Liouville-von-Neumann equations, ${ }^{17,18}$

$$
\frac{d}{d t} \rho=-\frac{1}{\hbar}\left[H_{0}+V, \rho\right]+\frac{d}{d t} \rho_{\text {diss }}
$$

where $\rho$ is the density operator and the dissipative term $d \rho_{\text {diss }} / d t$ describes the energy and phase relaxation via the coupling to the continuum of substrate excitations on a phenomenological basis. The Liouville-von-Neumann equations can be reduced to a set of three coupled differential equations using the assumption of rapid dephasing between two energy levels (phase relation between the photoexcited electron and its photohole) which, for a metallic volume state, is a common approximation. ${ }^{19}$ In cross-correlation measurements, the two distinguishable pulses ( $\mathrm{p}$ - and s-polarized) are able to excite two different intermediate states with different lifetimes. Therefore, the description of the three-level system has to be expanded to a four-level system. Under the assumption of nearly constant initial-state population, one can express the excitation in a cross-correlation measurement by two coupled differential equations. In this formalism, the two-photon-photoemission process is described by a classical rate equation model. This model is implemented in a fitting algorithm in order to extract the decay constant out of the experimentally obtained cross-correlation traces. Three parameters affect the shape of the simulated cross-correlation trace, namely the intermediate state lifetimes and the two parameters which include the transition matrix elements.

We used laser pulses at low fluence and peak power to avoid space-charge effects or highly excited electron distributions. We emphasize that the count rate is much lower than one electron per pulse. Therefore, we measure the relaxation of individual excited electronic states rather than the collective behavior of transiently heated nonequilibrium distribution. The laser system used was a mode-locked Ti:sapphire laser, pumped by about $7 \mathrm{~W}$ from a $\mathrm{cw}$ Nd:YVO3 diode laser. The system delivers transform-limited and sech2 temporally shaped pulses of up to $7 \mathrm{~nJ} /$ pulse with a pulse width (FWHM) of $<30 \mathrm{fs}$ and a repetition rate of $76 \mathrm{MHz}$. The wavelength can be tuned to a range of $770-830 \mathrm{~nm}$. The linearly polarized output of the Ti:sapphire was frequencydoubled in $0.2-\mathrm{mm}$-thick beta barium borate (BBO) crystal to produce UV pulses of $h \nu=3 \mathrm{eV}$. The pulses were split by a beam-splitter to equal intensity (pump and probe pulses), and one path could be delayed with respect to the other by a computer-controlled delay stage. Both beams were combined collinearly by a second beam-splitter and were focused at $45^{\circ}$ with respect to the surface normal of the sample.

The sample was mounted in an UHV chamber with a base pressure $<10^{-10}$ mbar. The photoemitted electrons were detected along the surface normal $\left(k_{\|}=0\right)$ by a cylindrical sector energy analyzer (CSA). In general, we use a pass energy of $4 \mathrm{eV}$, which leads to roughly $100 \mathrm{meV}$ resolution.

16.5-nm-thick polycrystalline Ta film was evaporated on the thin sheet of mica in an attached preparation chamber. Mica was cleaved on air shortly before evacuating and heated in UHV at $300^{\circ} \mathrm{C}$. Ta was directly evaporated from a 1.7-mm-thick rod (of $99.9 \%$ purity). The evaporation rate

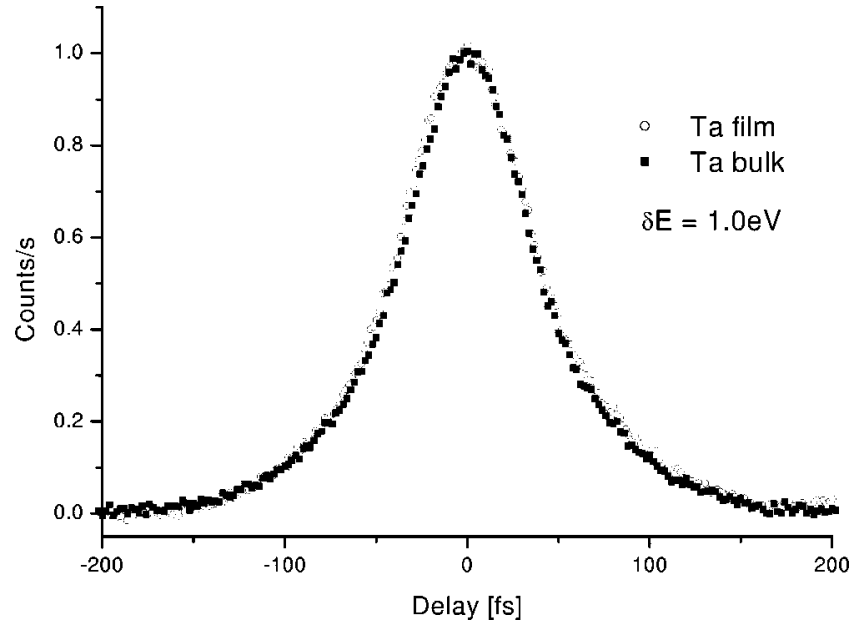

FIG. 1. 2PPE-autocorrelation traces obtained from polycrystalline Ta films $(\mathrm{FWHM}=91.8 \mathrm{fs})$ and bulk $(\mathrm{FWHM}=91.1 \mathrm{fs})$.

(around $1 \mathrm{ML} / \mathrm{min}$ ) was checked with a quartz oscillator, which is calibrated against atomic-force-microscope thickness measurements. In order to study the contribution due to the transport effect, a commercial polycrystalline Ta sheet was also cleaned for the measurement by a standard sputter procedure. To probe lower excited states, we reduced the work function by dosing the Ta surface with a small amount of Cs $(<0.1 \mathrm{ML})$.

As was shown in noble metals earlier, ${ }^{9}$ transport of optically excited electrons into the bulk can significantly affect the measured lifetime within a TR-2PPE experiment. Transport does play a role at low excitation energies and can even dominate the measured relaxation dynamics already at film thicknesses around $20 \mathrm{~nm}$. We performed a comparative experiment between polycrystalline Ta bulk and 16.5-nm-thick film (see Fig. 1). In the obtained cross-correlation traces, we found no clear indication of the carrier redistribution in the surface region within our temporal resolution of about $1 \mathrm{fs}$. Therefore, we can exclude any influence of the extracted lifetime in our Ta film due to electron transport.

In first-principles methods based on MBPT, ${ }^{12}$ an excited electron lifetime is calculated from the expectation value of the self-energy operator. In the GWA, ${ }^{12,20}$ one retains for the self-energy only the first-order term of the expansion in terms of a screened potential $W$,

$$
\Sigma(1,2)=i G(1,2) W(1,2),
$$

where $G$ is Green's function. We omit the spin coordinate and use shorthand notation $1 \equiv\left(\mathbf{r}_{1}, t_{1}\right)$. We extend the GW self-energy calculations by including high-order self-energy diagrams within the $T$-matrix approximation. ${ }^{20}$ Of all the terms of the $T$-matrix theory, we retain terms that describe multiple scatterings between electrons and holes. The $T$-matrix operator is defined as a solution of the BetheSalpeter equation,

$$
\begin{aligned}
T(1,2 \mid 3,4)= & W(1,2) \delta(1-3) \delta(2-4)+W(1,2) \\
& \times \int d 1^{\prime} d 2^{\prime} K\left(1,2 \mid 1^{\prime}, 2^{\prime}\right) T\left(1^{\prime}, 2^{\prime} \mid 3,4\right) .
\end{aligned}
$$

The kernel $K$ (electron-hole propagator) of this equation is a 
product of particle and hole Green functions,

$$
K\left(1,2 \mid 1^{\prime}, 2^{\prime}\right)=i G\left(1,1^{\prime}\right) G\left(2^{\prime}, 2\right) .
$$

The $T$-matrix contribution to the self-energy of a paramagnetic system is expressed as

$$
\Sigma(4,2)=-2 i \int d 1 d 3 G(3,1) T(1,2 \mid 3,4),
$$

where spin degeneracy has been included.

In order to make the calculations feasible, we employ the static approximation $W(1,2)=W\left(\mathbf{r}_{1}, \mathbf{r}_{2}\right) \delta\left(t_{1}-t_{2}\right)$ for the screened potential and local and static approximation, $1=2$, $3=4$, for the $T$-matrix operator. For the Green's function, we use the LDA noninteracting Green's function. First we calculate the spectral function of the kernel,

$$
\begin{aligned}
S(1,2, \omega)= & \sum_{\mathbf{k} n} \sum_{\mathbf{k}^{\prime} n^{\prime}}^{\text {occ unocc }}\left\{-\psi_{\mathbf{k} n}(1) \psi_{\mathbf{k}^{\prime} n^{\prime}}^{*}(1) \psi_{\mathbf{k}^{\prime} n^{\prime}}(2) \psi_{\mathbf{k} n}^{*}(2)\right. \\
& \times \delta\left(\omega+\epsilon_{\mathbf{k}^{\prime} n^{\prime}}-\epsilon_{\mathbf{k} n}\right) \\
& +\psi_{\mathbf{k} n}(1) \psi_{\mathbf{k}^{\prime} n^{\prime}}^{*}(1) \psi_{\mathbf{k}^{\prime} n^{\prime}}(2) \psi_{\mathbf{k} n}^{*}(2) \delta\left(\omega-\epsilon_{\mathbf{k}^{\prime} n^{\prime}}\right. \\
& \left.\left.+\epsilon_{\mathbf{k} n}\right)\right\} .
\end{aligned}
$$

Then the kernel is obtained through the Hilbert transform,

$$
-K(1,2, \omega)=\mathcal{P} \int d \omega^{\prime} \frac{S\left(1,2, \omega^{\prime}\right)}{\left(\omega-\omega^{\prime}\right)}-i \pi S(1,2, \omega) .
$$

In the frequency representation, the imaginary part of the $T$-matrix self-energy term for electrons is written as

$$
\begin{aligned}
\operatorname{Im} \Sigma(4,2, \omega)= & -2 \sum_{\mathbf{k}} \sum_{n^{\prime}}^{\text {unocc }} \int d(1) d(3) \psi_{\mathbf{k} n^{\prime}}(3) \psi_{\mathbf{k} n^{\prime}}^{*}(1) \\
& \times \operatorname{Im} T\left(1,2 \mid 3,4, \omega-\epsilon_{\mathbf{k} n^{\prime}}\right) \theta\left(\omega-\epsilon_{\mathbf{k} n^{\prime}}\right) .
\end{aligned}
$$

The real part of $\Sigma$ is also calculated through the Hilbert transform.

Having the calculated $T$-matrix self-energy, we add together the GWA and $T$-matrix self-energies and solve the Dyson equation for the complex quasiparticle energy $E_{\mathbf{q} n}(\omega)$,

$$
E_{\mathbf{q} n}(\omega)=\epsilon_{\mathbf{q} n}+\left\langle\psi_{\mathbf{q} n}|\Delta \Sigma(\omega)| \psi_{\mathbf{q} n}\right\rangle .
$$

Here $\Delta \Sigma(\omega)=\Sigma(\omega)-V^{x c}($ LDA $)$, with $V^{x c}($ LDA $)$ being the LDA exchange-correlation potential, provides many-body corrections to the LDA eigenvalues $\Delta \epsilon_{\mathbf{q} n}=E_{\mathbf{q} n}-\epsilon_{\mathbf{q} n}$. We solve the equation by employing the renormalization factor $Z,{ }^{12}$

$$
Z_{\mathbf{q} n}=\left(1-\frac{\partial \operatorname{Re} \Delta \Sigma_{\mathbf{q} n}(\omega)}{\partial \omega}\right)_{\omega=\epsilon_{\mathbf{q} n}}^{-1},
$$

and neglecting the imaginary part of $Z_{\mathbf{q} n}$. Finally, the imaginary part of $\Delta \epsilon_{\mathbf{q} n \sigma}$ determines the inverse of a quasiparticle lifetime

$$
\tau_{\mathbf{q} n}^{-1}=2 \times\left|\operatorname{Im} \Delta \epsilon_{\mathbf{q} n}\right|,
$$

where $\operatorname{Im} \Delta \epsilon_{\mathbf{q} n}=Z_{\mathbf{q} n} \times\left\langle\psi_{\mathbf{q} n}\left|\Delta \Sigma\left(\epsilon_{\mathbf{q} n}\right)\right| \psi_{\mathbf{q} n}\right\rangle$.

We perform LDA band-structure calculations within the

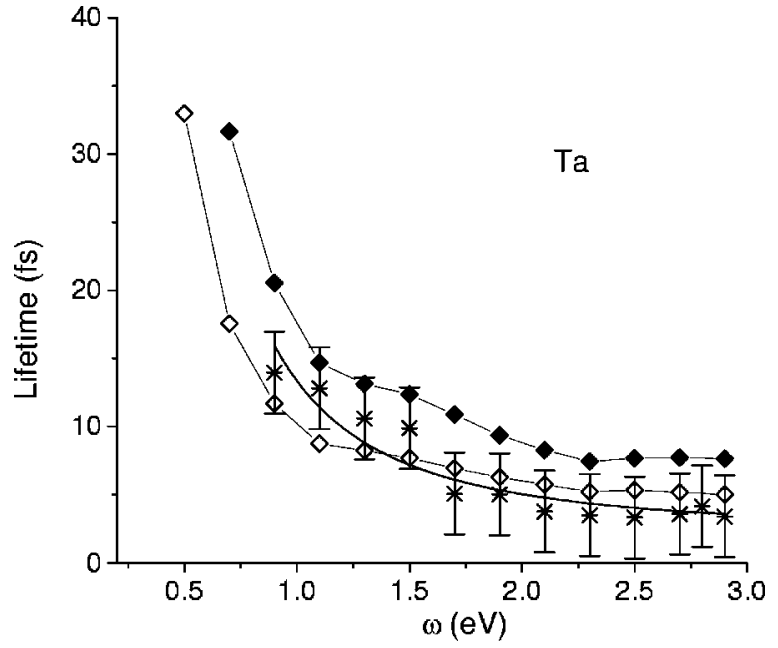

FIG. 2. Experimental and momentum-averaged electron lifetimes in Ta versus excitation energy $\omega=E-E_{F}$. Solid diamonds: theoretical averaged GW lifetimes; open diamonds: theoretical averaged GW $+\mathrm{T}$ lifetimes. Stars: experimental data, shown together with the error bars. Thick solid line is a guide for eye.

linear muffin-tin-orbitals (LMTO) method, ${ }^{21}$ and use the basis of LMTO product orbitals ${ }^{22}$ for many-body calculations. The screened potential $W$ is evaluated from first principles within the random-phase approximation, with the details of calculations similar to those for $\mathrm{Nb}, \mathrm{Mo}, \mathrm{Rh}$, and Pd. ${ }^{14}$ The details of the $T$-matrix calculations correspond to those published in Ref. 15. In particular, in the current discussion, we omit the so-called double-counting term which appears to be small in Ta.

Figure 2 shows the relaxation times of photoexcited electrons extracted from experimental cross-correlation traces and the theoretical momentum-averaged lifetimes as a function of energy $\omega=E-E_{F}$. We show the lifetimes up to the highest excited state probed in the experiment, $3.0 \mathrm{eV}$. The error bars for the experimental lifetime data are given by the statistical uncertainty of the detected photoelectrons as well as the exact determination of the laser pulse width in the UHV chamber. As expected for transition metals, ${ }^{7}$ the measured absolute lifetime values in the probed energy region lie below $15 \mathrm{fs}$. It is explained by the fact that, in comparison with electrons in noble metals, the density of occupied and unoccupied states near the Fermi level in Ta is much higher. So the available phase space for inelastic electron-electron scattering in Ta is considerably larger. This leads to a markedly bigger value of the GWA contribution to $\operatorname{Im} \Sigma$. Due to high density of states, we also have a noticeable contribution of the $T$-matrix term which decreases the GWA lifetimes by about $30-40 \%$.

Starting from highly excited states, we find a very good agreement between experimental and theoretical GW+T results, whereas the GW results are about 2 fs higher than the top limit of error bars. But the situation is different at the energy below $1.6 \mathrm{eV}$, when the $\mathrm{GW}+\mathrm{T}$ results are on the bottom limits of the error bars. At $1.6 \mathrm{eV}$, we do not find abrupt changes either in band structures or in characteristics defining the momentum-averaged lifetimes, such as density of states or convolution of density of states. ${ }^{14} \mathrm{We}$ suppose, 
therefore, that at the excitation energy below $1.6 \mathrm{eV}$, the cascade electrons refill underlying states, increasing in such a way the TR-2PPE signal. ${ }^{9}$ Qualitatively, the obtained results can be interpreted in terms of the evaluation by Quinn, who concluded that in a typical inelastic electron-electron collision, an excited electron loses about $\frac{2}{3}$ of its excitation energy. ${ }^{23}$ Ritchie found that this value decreases if one takes the antisymmetry of the electron wave function into account. ${ }^{24}$ These evaluations agree nicely with our results about the 30-40\% contribution of multiple scattering described by the $T$-matrix. In a similar way, one can explain the appearance of cascade electrons at low excitation energies. In our experiments, the highest excited state has an energy about $3 \mathrm{eV}$. So, following Quinn, cascade electrons influence mainly the population decay and, hence, the relaxation time of the excited states whose energy is less than $1 \mathrm{eV}$. According to Ritchie, this value can be bigger. Our estimation is $1.6 \mathrm{eV}$, although because of statistical uncertainty of the experimental results we cannot define it with high reliability. We would also like to emphasize that the increase in the specific lifetime due to cascade effects cannot be larger than the relaxation time of the higher energetic states where the electron originates.

In this Brief Report, we have presented experimental results on the relaxation times of excited electrons in Ta obtained by the TR-2PPE spectroscopy. We have also discussed the first-principles $\mathrm{GW}$ and $\mathrm{GW}+\mathrm{T}$-matrix calculations of excited electron lifetimes. We have found that the multiple scattering between holes and electrons included in the $T$-matrix produces noticeable contributions to the fast electrons deexcitation. At excitation energy above $1.6 \mathrm{eV}$, we find a very good agreement between experimental relaxation times and $\mathrm{GW}+\mathrm{T}$ theoretical lifetimes. Below $1.6 \mathrm{eV}$, the experimental relaxation times are higher than the theoretical data, which we refer to as the cascade effect of the electrons with high energy of excitation.

We acknowledge partial support by MCyT (Grant No. MAT 2001-0946) and the European FP6 Network of Excellence [FP6-NoE NANOQUANTA (500198-2)].
${ }^{1}$ R. Hight, Surf. Sci. Rep. 21, 275 (1995).

${ }^{2}$ C. A. Schmuttenmaer, M. Aeschlimann, H. E. Elsayed-Ali, R. J. D. Miller, D. A. Mantell, J. Cao, and Y. Gao, Phys. Rev. B 50, 8957 (1994).

${ }^{3}$ M. Aeschlimann, M. Bauer, and S. Pawlik, Chem. Phys. 205, 127 (1996).

${ }^{4}$ T. Hertel, E. Knoesel, M. Wolf, and G. Ertl, Phys. Rev. Lett. 76, 535 (1996).

${ }^{5}$ S. Ogawa, H. Nagano, and H. Petek, Phys. Rev. B 55, 10869 (1997).

${ }^{6}$ M. Aeschlimann, M. Bauer, S. Pawlik, W. Weber, R. Burgermeister, D. Oberli, and H. C. Siegmann, Phys. Rev. Lett. 79, 5158 (1997).

${ }^{7}$ R. Knorren, K. H. Bennemann, R. Burgermeister, and M. Aeschlimann, Phys. Rev. B 61, 9427 (2000).

${ }^{8}$ W. Nessler, S. Ogawa, H. Nagano, H. Petek, J. Shimoyama, Y. Nakayama, and K. Kishio, Phys. Rev. Lett. 81, 4480 (1998).

${ }^{9}$ M. Aeschlimann, M. Bauer, S. Pawlik, R. Knorren, G. Bouzerar, and K. H. Bennemann, Appl. Phys. A: Mater. Sci. Process. 71, 485 (2000).

${ }^{10}$ H.-J. Drouhin, Phys. Rev. B 62, 556 (2000).

${ }^{11}$ E. Zarate, P. Apell, and P. M. Echenique, Phys. Rev. B 60, 2326 (1999).

${ }^{12}$ G. D. Mahan, Many-Particle Physics (Plenum Press, New York, 1990).
${ }^{13}$ I. Campillo, V. M. Silkin, J. M. Pitarke, E. V. Chulkov, A. Rubio, and P. M. Echenique, Phys. Rev. B 61, 13484 (2000).

${ }^{14}$ V. P. Zhukov, F. Aryasetiawan, E. V. Chulkov, and P. M. Echenique, Phys. Rev. B 65, 115116 (2002).

${ }^{15}$ V. P. Zhukov, E. V. Chulkov, and P. M. Echenique, Phys. Rev. Lett. 93, 096401 (2004).

${ }^{16} \mathrm{R}$. Loudon, The Quantum Theory of Light (Oxford University Press, Oxford, 1973).

${ }^{17}$ M. Bauer, S. Pawlik, and M. Aeschlimann, Phys. Rev. B 60, 5016 (1999).

${ }^{18}$ S. Ogawa, J. Electron Spectrosc. Relat. Phenom. 124, 245 (2002).

${ }^{19}$ M. J. Weida, S. Ogawa, M. Nagano, and M. Petek, J. Opt. Soc. Am. B 17, 1443 (2000).

${ }^{20}$ A. L. Fetter and J. D. Walecka, Quantum Theory of ManyParticle Systems (McGraw-Hill, New York, 1971).

${ }^{21}$ O. K. Andersen, O. Jepsen, and M. Sob, in Electronic Band Structure and Its Applications, Vol. 283 of Lecture Notes in Physics, edited by M. Yussouff (Springer, Heidelberg, 1987).

${ }^{22}$ F. Aryasetiawan, in Strong Coulomb Correlations in Electronic Structure Calculations, edited by V. I. Anisimov (Gordon and Beach, Singapore, 2001).

${ }^{23}$ J. J. Quinn, Phys. Rev. 126, 1453 (1962).

${ }^{24}$ R. H. Ritchie, Phys. Rev. 114, 644 (1960). 